\title{
A Prototype Design for Medical Diagnosis by an Expert System
}

\author{
Syeda Huma Jabeen ${ }^{1,2+}$ and Gaoshou Zhai ${ }^{1}$ \\ ${ }^{1}$ School of Computer and Information Technology, Beijing Jiaotong University (BJTU), P. R. China \\ ${ }^{2}$ University of Engineering and Technology (UET), Lahore, 54000, Pakistan
}

\begin{abstract}
This prototype interface has been designed by keeping an eye on previously designed medical diagnose expert systems. The focus of this designed prototype interface is chronic diseases of remote areas due to which people mostly get suffered. So, in order to design that prototype Firstly, collect some data by some medical expert. Secondly, make a database by using SQL Lite. Thirdly, Interaction with the people is through user-friendly interfaces developed using Visual Studio 2010. Data elicited for the isolation, identification of symptoms and possible recommendations on susceptibility patterns makes the knowledge base which was modeled into frames at the different levels of the decision trees and using the "IF-THEN" production rules, quick deductions are made. This software makes inferences on the base of provided symptoms and gives the output in the form of the disease. Furthermore, click on the disease and its medicines, tests, and precautions, it will display on their alternative panel.
\end{abstract}

Keywords: Expert System, Knowledge Based Systems, Medical Diagnosis, E-Health.

\section{Introduction}

In an era of 1960, EXPERT systems (ES) were first developed by AI community. The expert system is a branch of artificial intelligence (AI) [1]. In Expert system, expertise are transferred from the doctors to computer. All kind of data or information is stored in computer and user can get all the information when it is needed. The system can provide a conclusion on the basis of stored information, it can make inferences and can provide a specific conclusion. The Expert system provides solutions to various problems on basis of stored information [2].

The expert system for the human medical problem is the knowledge-based system. Previously various expert system has been developed for human medical problems. First of all, INTERNIST-I was developed to diagnose diseases based on symptoms [3]. For bacterial infections and remedy MYCIN was developed [4], CADUCEUS was developed to improve the MYCIN [5] another example is PUFF developed for the diagnosis of lungs diseases [6]. These are some notable examples there are several other expert systems [7].

A medical expert system basically aims at the on time identification and cure of disease. It is based on the programs and knowledge. Information from the medical expert system is similar to the information provided by proficient in a specific area [8]. The knowledge of specialized doctor is very important for the development of a medical expert system. This is comprised of two phases. In initial phase data about diseases is recorded by organized meetings with the patients and doctors. While in the second phase is IF and THEN production rules. IF rule is based on disease symptoms and THEN part contain the disease $[9,10]$.

The aim of this approach is to facilitate the people of remote areas of developing the country by designed the expert system prototype interface healthcare software in order to resolve the problem of an inadequate number of health professionals or doctors in remote areas.

\section{Methodology of Expert System}

\footnotetext{
+ Corresponding author. Tel.: : + 8615801393301.

E-mail address: hjabeen2@gmail.com; 15129127@bjtu.edu.cn.
} 
This expert system prototype design was developed by data acquisition through the various system. The system is a knowledge-based system as data was obtained from physicians, books and from journals $[4,6,11$, 12, and 13]. The process of design is described below.

\subsection{Principle of Prototype Design}

The system, developed as a data-driven prototype, went through several iterations and finally validated. The circumstances of expert system application are identified, refined with sub-conditions and then the various categories of investigations are formulated and classified according to the level of significance. The system is implemented in C\# runtime environment and visual studio 2010 for the various interfaces using the traditional life cycle system and the use case evolutionary model process. The system is easy to use and provides consistently reliable advice. In order to log on, there is a need for a correct username and password. There are log off buttons at the various system screens in case the user wants to close the program (Fig. 1).

\subsection{Architecture of Expert System Prototype}

The architecture of the expert system usually consists of inference engine (IE), knowledge base design, user interface, knowledge acquisition unit and explanatory module [4, 13] (Fig. 2).

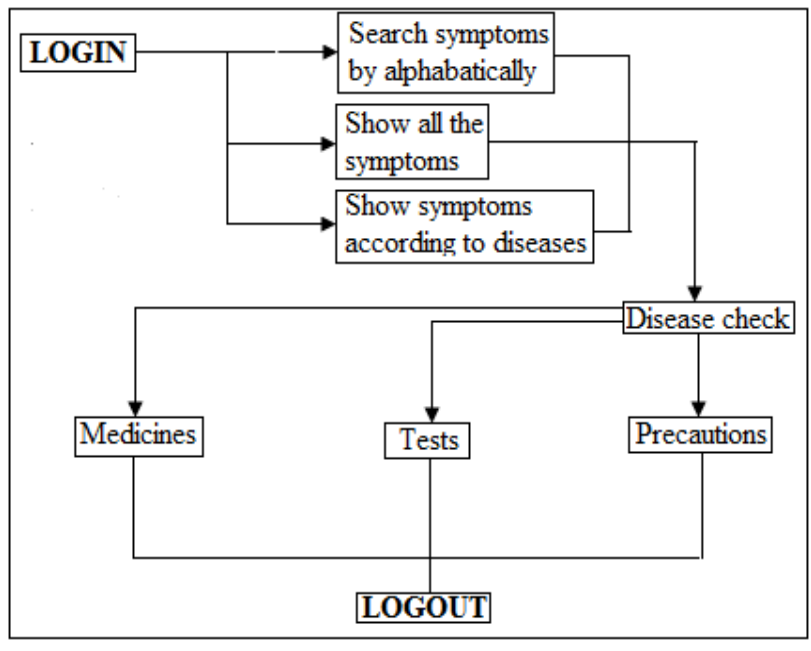

Fig. 1: Working principle of medical diagnose interface

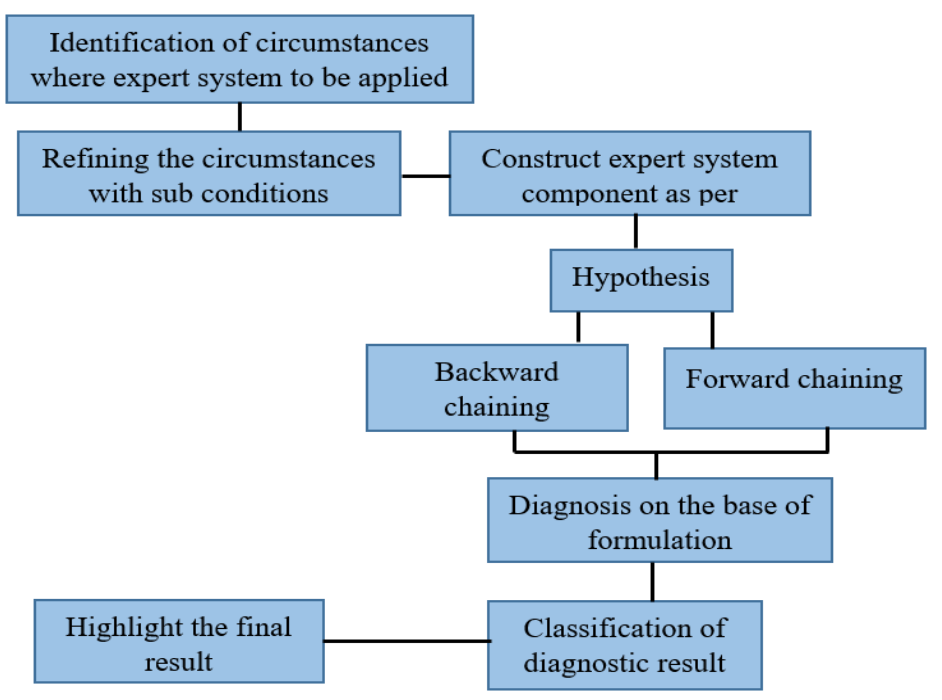

Fig. 2: Architecture of medical diagnose interface

\subsubsection{Inference Engine}

The inference engine is based on forward and backward chaining, examining the knowledge base (disease symptoms) for information that matches the user's query (kind of disease).

\subsubsection{Knowledge Base Design}

The knowledge domain was got from facts of a collection of data about the types of symptoms and diseases to be isolated and identified, the identification methods, the expected results. Data elicited for the isolation, identification of symptoms and possible recommendations on susceptibility patterns makes the knowledge base which was modeled into frames at the different levels of the decision trees and using the "IF-THEN" production rules, quick deductions are made (Fig. 3)

\subsubsection{User Interface Unit}

This unit allows a non-expert user to inquire (question) the expert system, and to find conclusion or advice. The user-interface is always designed as simple as it could be.

\subsubsection{Knowledge Acquisition Unit}

It is a process of acquiring knowledge from different sources mean acquiring, organizing, analyzing and studying of knowledge before applying. We obtained and study data bout various disease.

\subsubsection{Explanation Module}

This section usually enables a knowledge worker to why this information have been explained and why a specific fact is desired.

\section{The Prototype Design Functionalities}


The expert system was implemented as a prototype. Basing on the various interfaces below, a systematic step-by-step approach is demonstrated, starting from the login tab to the final medical diagnosis system.

\subsection{Prototype Interface Design}

\subsubsection{Home Page Interface}

The home page of software contains the various icons bar, search bar, Symptoms section and output text field which linked it directly to other pages. By clicking of any of it then the corresponding page of that will be open. The home page is the main page of software which contains icon bar, search bar, symptoms panel, and output text fields. (Fig 4).

\subsubsection{Hypothesis for Designed Prototype}

Generation of hypothesis processes is pervasive in human based reasoning. Hypothesis contains the data about disease detail which the system will be able to diagnose and give recommendations. In this part, medicines, symptoms, test name and precautions are assign to related disease. This is done through forward and backward chaining. The green arrows are used to allocate and de-allocate the require data to diseases. Save icon used to save the changes according to the requirements. In the process of allocation, first, we select the disease from the data field of the disease name then choose the corresponding symptoms, medicine name, test name, and precautions from their correspond data fields. After that, we click on the forward arrow, selected data moves in output data field and show all the required output. At last, we click on a save icon and then save the selected data. In this way, the selected data is assigned to disease (Fig. 5).

\subsubsection{Functionality of Interface Tables}

The home page of the interface have further icons over it by clicking on it will directly lead to corresponding page i.e Symptom, disease, precaution, and medicines. Each page shows the text field and table form in which can edit or delete the data directly without accessing the database in the SQL lite. It contains the database of all symptoms, diseases, precaution and medicines saves in SQL lite.

\section{Working of Prototype Interface for Disease Diagnosis.}

This system provides information to non-professionals. In the process of diagnosing of diseases, first of all, it will check the symptoms that explained by the patient. The software makes inferences on basis of symptoms and provides the output (disease). Furthermore, click on the disease and its medicines, tests, and precautions, it will display on their alternative panel. Such as medicine data list contain on all the medicines that are referred to the software according to resultant disease. The Same action will be done in the case of tests and precautions. This panel consists of four data list named as a disease, medicine, test, and precautions. The output that we received is in four forms disease, medicine, test, and precaution. If we received all the outputs in the single data list, it creates a mess. To avoid this phenomenon, we distinguish them in different data lists. This will also provide us the better view of output and easier to understand. Because of this, the physician can better explain the prescription to the patient (Fig. 6).

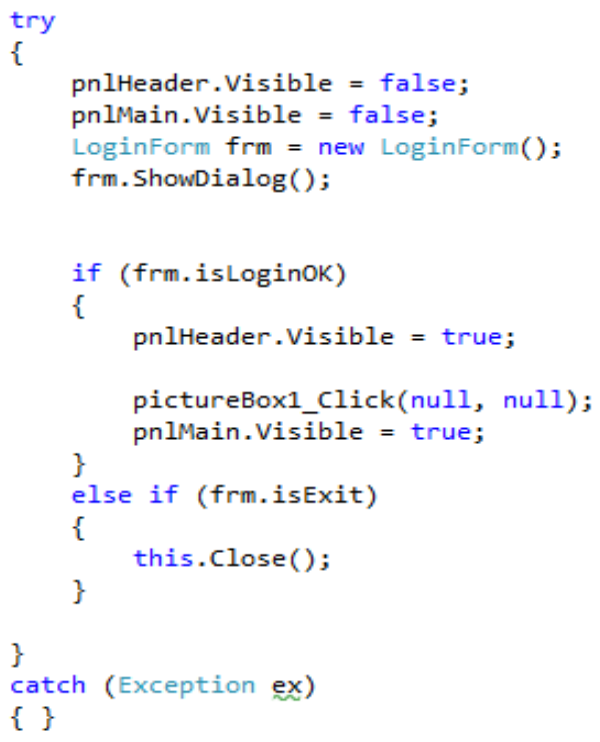

Fig. 3: IF-THEN production rules

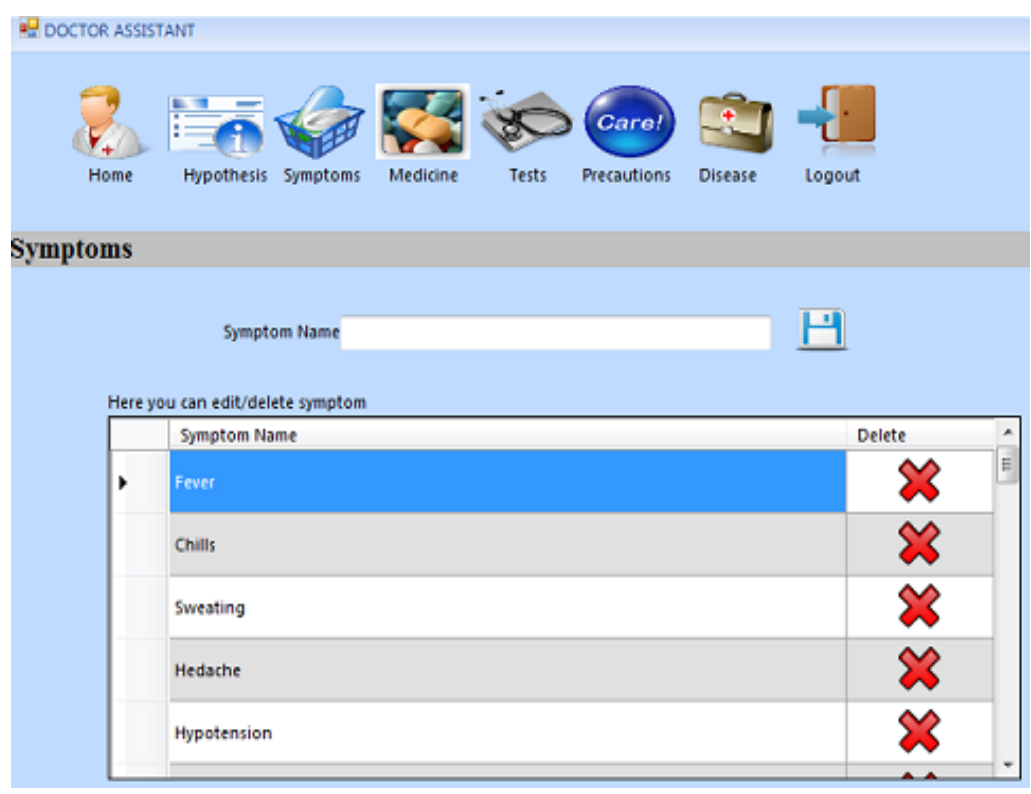

Fig. 4: Home page interface 


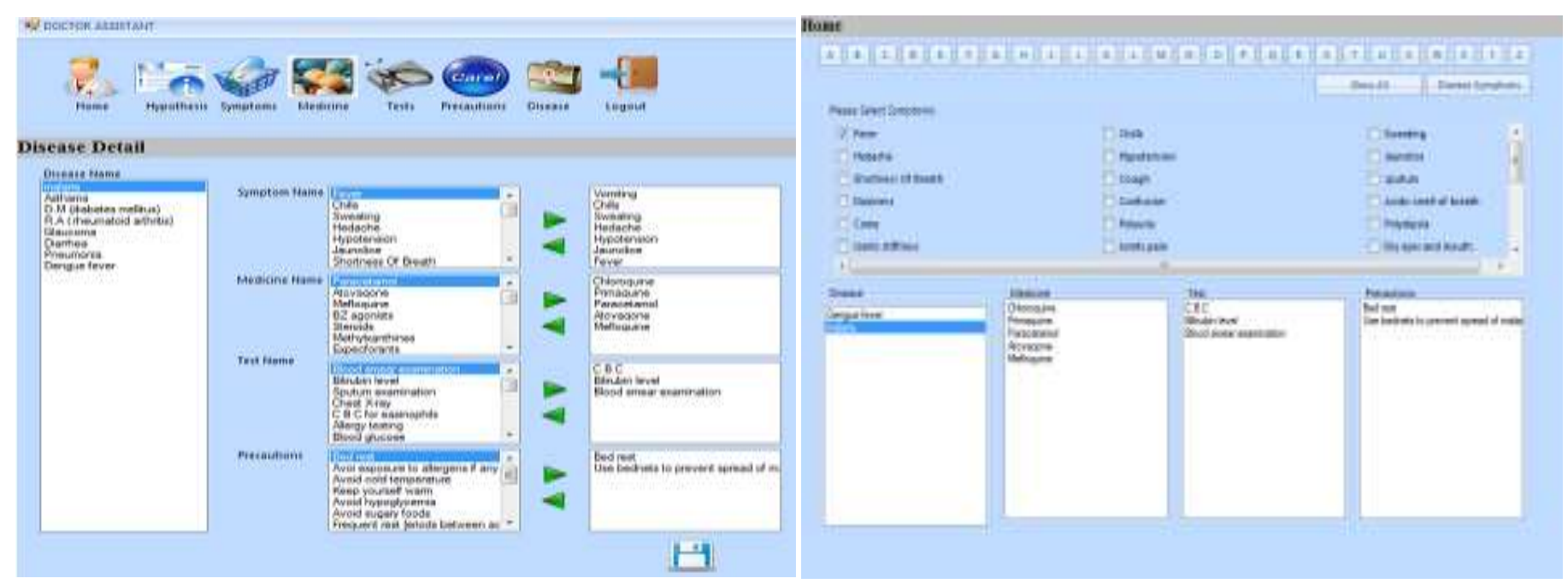

Fig. 5: Disease detail

Fig. 6: Result Disease Diagnosis

\section{Conclusion and Future Work}

The expert system prototype interface can bring the betterment in the health care of remote areas of developing countries by fulfilling the shortage of doctors. Our method can be used to develop a complete knowledge base medical diagnosis expert system to deal with the chronic disease of remote areas.

This study is a prototype implementation of an expert system for definitive isolation and identification of diseases, particularly in the tropical environments. The knowledge domain is got by extensive consultations with knowledge experts in the field of medicines and literature review. These consultations enabled the formulation of production rules. The expert system has been developed from scratch using $\mathrm{C \#}$ runtime environment. The process takes time but gives freedom to the developer to think and produce original work. Expert systems exercise information technology to acquire and utilize human expertise. They can be beneficial to organizations that have clear objectives, rules, and procedures by providing answers to repetitive decision-making, processes, and tasks. They can also reduce employment costs, centralize the decision-making process, create efficiency and reduce the time needed to solve complex problems. However, the weaknesses of these systems should never be under looked such as the limited knowledge domain compared to human experts.

\section{Acknowledgements}

We are thankful to Professor Xuezhong Zhou (Beijing Jiaotong Univeristy, BJTU) for reviewing the paper and giving useful comments for improving the manuscripts.

\section{References}

[1] S. J. Russell, P. Norvig, J. F. Canny, J. M. Malik, and D. D. Edwards. Artificial intelligence: a modern approach. Vol. 2. Upper Saddle River: Prentice hall, 2003.

[2] B. G. Hope and R. H. Wild. "An expert support system for service quality improvement." In System Sciences, 1994. Proceedings of the Twenty-Seventh Hawaii International Conference on, vol. 3, pp. 183-190. IEEE, 1994.

[3] R. A. Miller, H. E. Pople Jr, and J. D. Myers. "Internist-I, an experimental computer-based diagnostic consultant for general internal medicine." New England Journal of Medicine 307, no. 8 (1982): 468-476.

[4] W. R. Swartout. "Rule-based expert systems: The MYCIN experiments of the stanford heuristic programming project: BG Buchanan and EH Shortliffe, (Addison-Wesley, Reading, MA, 1984); (1985): 364-366.

[5] H. Pople. "Evolution of an expert system: from internist to caduceus." Artificial Intelligence in Medicine (1985): $179-208$.

[6] J. S. Aikins, John C. Kunz, E. H. Shortliffe, and R. J. Fallat. "PUFF: an expert system for interpretation of pulmonary function data." Computers and biomedical research 16, no. 3 (1983): 199-208.

[7] Mrs. S. S. Gulavani, and R. V. Kulkarni. "A Review of Knowledge Based Systems in Medical Diagnosis." International Journal of Information Technology and Knowledge Management 2, no. 2 (2009): 269275. 
[8] J. Singla. "The Diagnosis of Some Lung Diseases in a Prolog Expert System." International Journal of Computer Applications 78, no. 15 (2013).

[9] S. S. Abu Naser and A. Zaiter A. Ola. "An Expert System for Diagnosing Eye Diseases Using Clips." Journal of Theoretical \& Applied Information Technology 4, no. 10 (2008).

[10] Ibrahim M. Ahmed, Marco Alfonse, Mostafa Aref, and Abdel-Badeeh M. Salem. "Reasoning Techniques for Diabetics Expert Systems." Procedia Computer Science 65 (2015): 813-820.

[11] M. O. Yinyeh, and S. Alhassan. "Expert Health Diagnosis and Monitoring System Software for Common Medical Conditions." International Journal of Computer Applications 124, no. 1 (2015).

[12] P. S. K. Patra, D. P. Sahu, and I. Mandal. "An expert system for diagnosis of human diseases." International Journal of Computer Applications 1, no. 13 (2010): 71-73.

[13] Hudson, D. L., 2006. Medical expert systems. Wiley Encyclopedia of Biomedical Engineering. 